\title{
Direct optical transitions at K- and H-point of Brillouin zone in bulk $\mathrm{MoS}_{2}, \mathrm{MoSe}_{2}, \mathrm{WS}_{2}$, and $\mathrm{WSe}_{2}$
}

J. Kopaczek, M. P. Polak, P. Scharoch, K. Wu, B. Chen, S. Tongay, and R. Kudrawiec

Citation: Journal of Applied Physics 119, 235705 (2016); doi: 10.1063/1.4954157

View online: $\mathrm{http}: / / d x . d o i . o r g / 10.1063 / 1.4954157$

View Table of Contents: http://aip.scitation.org/toc/jap/119/23

Published by the American Institute of Physics

\section{Articles you may be interested in}

Materials properties of out-of-plane heterostructures of $\mathrm{MoS}_{2}-\mathrm{WSe}_{2}$ and $\mathrm{WS}_{2}-\mathrm{MoSe}_{2}$

Applied Physics Letters 108, 063105 (2016); 10.1063/1.4941755

Optical constants and dynamic conductivities of single layer $\mathrm{MoS}_{2}, \mathrm{MoSe}_{2}$, and $\mathrm{WSe}_{2}$

Applied Physics Letters 107, 083103 (2015); 10.1063/1.4929700

Low-temperature photocarrier dynamics in monolayer $\mathrm{MoS}_{2}$

Applied Physics Letters 99, 102109 (2011); 10.1063/1.3636402

The indirect to direct band gap transition in multilayered $\mathrm{MoS}_{2}$ as predicted by screened hybrid density functional theory

Applied Physics Letters 99, 261908 (2011); 10.1063/1.3672219

$\mathrm{H}$-point exciton transitions in bulk $\mathrm{MoS}_{2}$

Applied Physics Letters 106, 182103 (2015); 10.1063/1.4920986

2D-2D tunneling field-effect transistors using $\mathrm{WSe}_{2} / \mathrm{SnSe}_{2}$ heterostructures

Applied Physics Letters 108, 083111 (2016); 10.1063/1.4942647

\section{A|P| | Journal of}

Save your money for your research.

It's now FREE to publish with us no page, color or publication charges apply.
Publish your research in the

Joumal of Applied Physics

to claim your place in applied

physics history. 


\title{
Direct optical transitions at K- and H-point of Brillouin zone in bulk $\mathrm{MoS}_{2}$, $\mathrm{MoSe}_{2}, \mathrm{WS}_{2}$, and $\mathrm{WSe}_{2}$
}

\author{
J. Kopaczek, ${ }^{1, a)}$ M. P. Polak, ${ }^{1, a)}$ P. Scharoch, ${ }^{1}$ K. Wu, ${ }^{2}$ B. Chen, ${ }^{2}$ S. Tongay, ${ }^{2}$ \\ and R. Kudrawiec ${ }^{1, b)}$ \\ ${ }^{1}$ Faculty of Fundamental Problems of Technology, Wroclaw University of Technology, Wybrzeze \\ Wyspiańskiego 27, 50-370 Wrocław, Poland \\ ${ }^{2}$ School for Engineering of Matter, Transport and Energy, Arizona State University, Tempe, \\ Arizona 85287, USA
}

(Received 15 March 2016; accepted 5 June 2016; published online 21 June 2016)

\begin{abstract}
Modulated reflectance (contactless electroreflectance (CER), photoreflectance (PR), and piezoreflectance (PzR)) has been applied to study direct optical transitions in bulk $\mathrm{MoS}_{2}, \mathrm{MoSe}_{2}$, $\mathrm{WS}_{2}$, and $\mathrm{WSe}_{2}$. In order to interpret optical transitions observed in CER, PR, and PzR spectra, the electronic band structure for the four crystals has been calculated from the first principles within the density functional theory for various points of Brillouin zone including $\mathrm{K}$ and $\mathrm{H}$ points. It is clearly shown that the electronic band structure at $\mathrm{H}$ point of Brillouin zone is very symmetric and similar to the electronic band structure at $\mathrm{K}$ point, and therefore, direct optical transitions at $\mathrm{H}$ point should be expected in modulated reflectance spectra besides the direct optical transitions at the $\mathrm{K}$ point of Brillouin zone. This prediction is confirmed by experimental studies of the electronic band structure of $\mathrm{MoS}_{2}, \mathrm{MoSe}_{2}, \mathrm{WS}_{2}$, and $\mathrm{WSe}_{2}$ crystals by CER, PR, and PzR spectroscopy, i.e., techniques which are very sensitive to critical points of Brillouin zone. For the four crystals besides the $\mathrm{A}$ transition at $\mathrm{K}$ point, an $\mathrm{A}_{\mathrm{H}}$ transition at $\mathrm{H}$ point has been observed in CER, PR, and PzR spectra a few tens of meV above the A transition. The spectral difference between $\mathrm{A}$ and $\mathrm{A}_{\mathrm{H}}$ transition has been found to be in a very good agreement with theoretical predictions. The second transition at the $\mathrm{H}$ point of Brillouin zone $\left(\mathrm{B}_{\mathrm{H}}\right.$ transition) overlaps spectrally with the $\mathrm{B}$ transition at $\mathrm{K}$ point because of small energy differences in the valence (conduction) band positions at $\mathrm{H}$ and $\mathrm{K}$ points. Therefore, an extra resonance which could be related to the $\mathrm{B}_{\mathrm{H}}$ transition is not resolved in modulated reflectance spectra at room temperature for the four crystals. Published by AIP Publishing.
\end{abstract}

[http://dx.doi.org/10.1063/1.4954157]

\section{INTRODUCTION}

Transition metal dichalcogenides (TMDs), such as $\mathrm{MoS}_{2}, \mathrm{MoSe}_{2}, \mathrm{WS}_{2}$, and $\mathrm{WSe}_{2}$, have been very intensively investigated for the past few years since their electronic band structure and optical properties vary very strongly with the number of monolayers. ${ }^{1-3}$ The electronic band structure of bulk TMDs (mainly $\mathrm{MoS}_{2}$ ) has been investigated both in the past $^{4-11}$ and quite recently when researchers studied the evolution of the electronic band structure in these crystals with reduction of their sizes from a bulk regime through a few layers to a monolayer. ${ }^{12-14}$ However, some issues for bulk materials are still unclear. One of them is the electronic band structure at the $\mathrm{H}$ point of the Brillouin zone and optical transitions at this point. The space group $\mathrm{P}_{3} / \mathrm{mmc}$, to which all the studied crystals belong, has a Brillouin zone which is a hexagonal prism, as shown in Fig. 1, where two planes can evidently be distinguished: the one with the $\Gamma, \mathrm{M}$, and $\mathrm{K}$ high symmetry points and the one above it with $\mathrm{A}, \mathrm{L}$, and $\mathrm{H}$ points. However, since the main focus in the recent studies of these materials are monolayers, where the reciprocal

\footnotetext{
a) J. Kopaczek and M. P. Polak contributed equally to this work.

b) Author to whom correspondence should be addressed. Electronic mail: robert.kudrawiec@pwr.edu.pl
}

space is one dimensional and only the $\Gamma \mathrm{M} \mathrm{K}$ path is present, while comparing the band structures of monolayers and bulk materials, researchers tend to focus only on the $\Gamma \mathrm{M} \mathrm{K}$ path in the bulk as well. However, it seems obvious that there is a high similarity in $\Gamma \mathrm{M} \mathrm{K}$ points and $\mathrm{A} \mathrm{L} \mathrm{H}$ points, so they all should be considered in interpretation of the experimental spectra. Recently, Saigal and Ghosh ${ }^{15}$ studied bulk $\mathrm{MoS}_{2}$ by photoreflectance (PR) and have proposed that excitonic transitions at the $\mathrm{H}$ point of Brillouin zone are observed $\sim 30 \mathrm{meV}$ above excitonic transitions at the $\mathrm{K}$ point of Brillouin zone. The authors have concluded about such interpretation of PR spectra on the basis of detailed lineshape analysis of PR resonances without theoretical calculations of

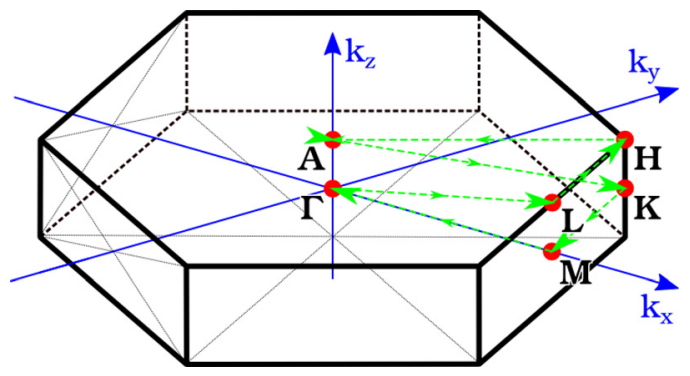

FIG. 1. Brillouin zone of $2 \mathrm{H}-\mathrm{MX}_{2}(\mathrm{M}=\mathrm{Mo}$ or $\mathrm{W}$ and $\mathrm{X}=\mathrm{S}$ or $\mathrm{Se})$. 
the electronic band structure for this crystal. Previous studies of $\mathrm{MoS}_{2}$ crystals by modulation spectroscopy ${ }^{16-19}$ have not focused on this aspect. Moreover, this issue has never before been studied either experimentally or theoretically for $\mathrm{MoSe}_{2}, \mathrm{WS}_{2}$, and $\mathrm{WSe}_{2}$ crystals. It is worth noting that the electronic band structure for bulk $\mathrm{MoS}_{2}$ was reported in many papers, but the $\mathrm{H}$ point of Brillouin zone is usually neglected in theoretical analysis, and therefore, it is still unclear how important are optical transitions at this point of Brillouin zone in the interpretation of PR spectra or other differential absorption-like spectra such as contactless electroreflectance (CER) or piezoreflectance (PzR). So far, CER spectroscopy has never been applied to study TMDs but PzR spectroscopy was widely applied to study bulk TMDs. ${ }^{20-24}$ However, optical transitions at the $\mathrm{H}$ point of Brillouin zone have not been identified in these spectra. It means that the issue of optical transitions at the $\mathrm{H}$ point of Brillouin zone is an open question for bulk TMDs, especially that this issue has never been considered for TMDs other than $\mathrm{MoS}_{2}$ (i.e., $\mathrm{MoSe}_{2}, \mathrm{WS}_{2}$, and $\mathrm{WSe}_{2}$ ).

In this work, the electronic band structure for $\mathrm{MoS}_{2}$, $\mathrm{MoSe}_{2}, \mathrm{WS}_{2}$, and $\mathrm{WSe}_{2}$ crystals is calculated from the first principles within the density functional theory (DFT) for various points of Brillouin zone including $\mathrm{H}$ point. It is clearly shown that the electronic band structure at $\mathrm{H}$ point is very symmetric, and hence, a strong optical transition at this point of Brillouin zone is expected in absorption-like spectra. This prediction is confirmed by experimental studies of the electronic band structure of $\mathrm{MoS}_{2}, \mathrm{MoSe}_{2}, \mathrm{WS}_{2}$, and $\mathrm{WSe}_{2}$ crystals by contactless electroreflectance (CER), photoreflectance, and piezoreflectance, i.e., modulation spectroscopy which is very sensitive to critical points (CP) of Brillouin zone. This paper is organized as follows. In Section II, details on the electronic band structure calculations and CER, PR, and PzR experiments are given. Results of DFT calculations of the electronic band structure for $\mathrm{MoS}_{2}, \mathrm{MoSe}_{2}, \mathrm{WS}_{2}$, and $\mathrm{WSe}_{2}$ crystals and results of measurements of CER, PR, and PzR spectra for these crystals together with their interpretation and discussion are given in Section III. Conclusions from our studies are summarized in Section IV.

\section{METHODS}

\section{A. Band structure calculations}

The DFT calculations of $\mathrm{MoS}_{2}, \mathrm{MoSe}_{2}, \mathrm{WS}_{2}$, and $\mathrm{WSe}_{2}$ bulk crystals have been performed with the full-potential linearized augmented plane wave method, as implemented in the WIEN2k code. ${ }^{25}$ The van der Waals interactions, very important in geometry optimization of this type of systems, have been included via the DFT-D3 dispersion correction as proposed by Grimme et al. ${ }^{26}$ used together with generalized gradient approximation (GGA) (PBE) functional. ${ }^{27}$ This approach provides an excellent agreement of the geometry optimization with the experimental values (less than $1 \%$ of discrepancy), as we have proven in our previous studies ${ }^{28}$ where a detailed comparison of different approaches and functionals can be found. To improve the description of the band structure of regular LDA/GGA approach, we have used the modified Becke-Johnson exchange potential with LDA correlation
(MBJLDA) $)^{29,30}$ for all our band structure calculations. This approach has been proven by us to be very effective and efficient numerous times over the years in more traditional III-V semiconductors $^{31,32}$ as well as in TMDs in Ref. 28 where a detailed reasoning of this choice is presented. A $10 \times 10 \times 6$ Monkhorst-Pack mesh was used, as a result of convergence studies. The basis set was determined by RKmax equal to 9, and the atomic sphere radii of 2.35, 2.42, 2.02, and 2.3 bohrs were used for Mo, W, S, and Se, respectively.

\section{B. Samples}

$\mathrm{MoS}_{2}, \mathrm{MoSe}_{2}, \mathrm{WS}_{2}$, and $\mathrm{WSe}_{2}$ crystals were grown by iodine assisted vapor transport technique at high temperatures $\left(900-1100^{\circ} \mathrm{C}\right)$ and low pressures $\left(\sim 1 \times 10^{-6}\right.$ Torr $)$ in a sealed quartz ampoules. During growth at $\sim 50^{\circ} \mathrm{C}$, temperature differential created between hot and cold zones to initiate nucleation and facilitate precursor transport. Prior to growth, quartz ampoules $(\sim 15 \mathrm{~cm}$ in length, $2.4 \mathrm{~cm}$ outer diameter, 2.0 inner diameter) were cleaned in piranha solution and annealed in $\mathrm{H}_{2}$ gas to remove contaminants. Precursors (Mo, W foils and S, Se nuggets) were mixed in 1:2.05 M:X stoichiometric ratio, and iodine pieces were added as a transport agent. Quartz ampoule is sealed under vacuum ( $1 \mu$ Torr). Samples used to CER, PR, and PzR studies were flakes of macroscopic sizes: $\sim 3 \times 3 \mathrm{~mm}$ in hexagonal plane and thickness $<0.2 \mathrm{~mm}$ perpendicular to the hexagonal plane. In these measurements, white light reflects at near-normal incidence from the hexagonal plane of the sample.

\section{Contactless electroreflectance, photoreflectance, and piezoreflectance measurements}

CER, PR, and PzR measurements have been performed in the so-called "bright configuration" ${ }^{33}$ where the sample was illuminated by a spectrum of white light from a halogen lamp $(150 \mathrm{~W})$ at normal incidence. Next, the light reflected from the sample is dispersed through a $0.55 \mathrm{~m}$ focal length single grating monochromator and detected by $\mathrm{Si}$ photodiode. The signal measured by photodiode has two components: (i) the DC component which is proportional to $\mathrm{I}_{0} \mathrm{R}$ and (ii) the $\mathrm{AC}$ component which is proportional to $\mathrm{I}_{0} \Delta \mathrm{R}$. The change in the reflectance spectrum $(\Delta R)$ appears due to modulation of the built-in electric field (CER and PR measurements) or strain (PzR case) inside the sample. Both DC and AC components are measured with a lock-in amplifier $\left(\mathrm{I}_{0}\right.$ is the intensity of reflected light). A computer divides the AC signal by the DC component giving the $\Delta R / R(E)$ spectrum, where $E$ is the photon energy of the incident beam. Phase-sensitive detection of the $\Delta \mathrm{R}$ signal allows to eliminate the background signal and detect even very weak optical transitions which usually are not observed in the reflectance spectra at room temperature.

For CER measurements, the samples were placed in a capacitor with a half-transparent top electrode made from a copper-wire mesh. ${ }^{34}$ They were glued to the bottom copper electrode by a silver paste. The distance between the sample surface and the top electrode was $\sim 0.5 \mathrm{~mm}$. Thus, there is nothing in direct contact with the sample. It means that the sample does not conduct any currents, and the external electric field is able to change the carrier distribution inside the 
sample. Note that the main drop in voltage in this system appears in the air gap between the front electrode and the sample. The limit for the applied voltage is the electric breakdown in this air gap. A generator of square AC voltage made in-house was used to generate the AC field inside the capacitor. A maximum peak-to-peak alternating voltage of $\sim 3.5 \mathrm{kV}$ with the frequency of $280 \mathrm{~Hz}$ was used for the modulation.

The pump beam for PR measurements was provided by the $405 \mathrm{~nm}$ line of a semiconductor laser. Its intensity was $\sim 50 \mathrm{~mW}$ and diameter on the sample was $\sim 3 \mathrm{~mm}$. The laser light was chopped by a mechanical chopper with a frequency of $280 \mathrm{~Hz}$.

For PzR measurements, samples were glued on piezoceramics by acrylic glue. AC voltage with an amplitude of $300 \mathrm{~V}$ and the frequency of $280 \mathrm{~Hz}$ was applied to this piezoceramics in order to modulate strain inside the sample.

The periodic perturbation of the built-in electric field (CER and PR measurements) and strain (piezoreflectance) inside the sample is very small which is the principle of modulation spectroscopy. However, this perturbation is sufficient to slightly change the parameters of an optical transition (its energy, broadening, and intensity) and generate changes in reflectance spectrum which are detected in the lock-in technique.

\section{RESULTS AND DISCUSSION}

The electronic band structure for $\mathrm{MoS}_{2}, \mathrm{MoSe}_{2}, \mathrm{WS}_{2}$, and $\mathrm{WSe}_{2}$ crystals calculated from the first principles with the GGA+DFT-D3 geometry optimization and MBJLDA band structure is given in Fig. 2. As mentioned earlier, to properly interpret the transitions observed in experimental measurements, considerations of band structures throughout the whole Brillouin zone, with all the high symmetry points included, are extremely important. Hence, our band structure plots have been calculated along the $\mathrm{A} \rightarrow \mathrm{K} \rightarrow \mathrm{M} \rightarrow \mathrm{\Gamma} \rightarrow \mathrm{L}$ $\rightarrow \mathrm{H} \rightarrow \mathrm{A}$ path, as shown in Fig. 1. The band gap for the four crystals is indirect. Its conduction band minimum and valence band maximum are located at $\Lambda$ and $\Gamma$ points, respectively, see Fig. 2. Modulation spectroscopy is not sensitive to indirect optical transitions, so the indirect gap is not probed by $\mathrm{CER}, \mathrm{PR}$, or PzR spectroscopy. These techniques are, however, very sensitive to direct optical transitions at critical points of Brillouin zone, and therefore, all points of high symmetry have to be taken into account when the modulated reflectance spectra are analyzed and interpreted.

Probability of an interband transition in absorption $\left(W_{\vec{k}}\right)$ is given by Fermi's Golden Rule ${ }^{35,36}$

$$
W_{\vec{k}}=\frac{2 \pi}{\hbar}\left|\left\langle\psi_{c}\left|H^{\prime}\right| \psi_{v}\right\rangle\right|^{2} \rho_{c v}(\hbar \omega) .
$$

Here, $\mathrm{H}^{\prime}$ is the perturbing Hamiltonian for electromagnetic interaction, $\hbar$ is the Planck's constant, $\psi_{c}$ and $\psi_{v}$ are the unperturbed wave functions of the conduction and valence band states, respectively, and $\rho_{c v}$ is the joint density of states (JDS) which is given as

$$
\rho_{c v}(\hbar \omega)=\frac{1}{4 \pi^{2}} \int_{S(E)} \frac{d S}{\left|\nabla_{k}\left(E_{c}-E_{v}\right)\right|},
$$

where $\mathrm{S}(\mathrm{E})$ is a constant energy surface such as $E=\hbar \omega$ $=E_{c}-E_{v}$. For direct optical transitions, a negligible change in the crystal momentum is assumed. Strong optical transitions will appear in modulated reflectance spectra (PzR, PR,
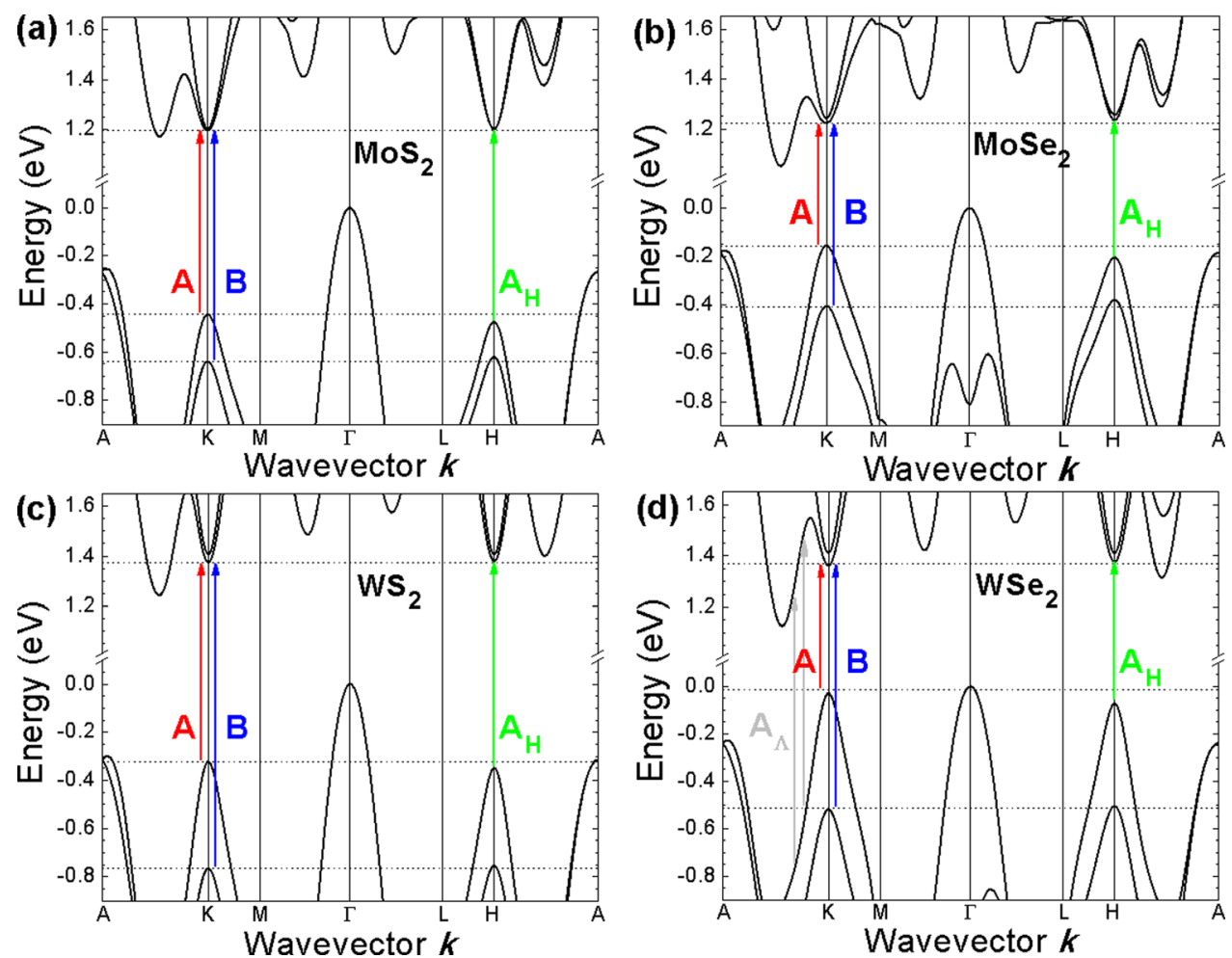

FIG. 2. Electronic band structure of bulk $\mathrm{MoS}_{2}$ (a), $\mathrm{MoSe}_{2}$ (b), WS 2 (c), and $\mathrm{WSe}_{2}$ (d) at various points of Brillouin zone. Vertical arrows represent direct optical transitions observed in modulated reflectance spectra. 
and CER $)$ at energies where $\nabla_{k}\left(E_{c}-E_{v}\right) \approx 0$. Such points are called critical points (CP) and they can be of several types. ${ }^{37,38}$ If $\nabla_{k} E_{c}=\nabla_{k} E_{c}=0$, we have either a minimum, a maximum, or a saddle point in each band; this usually occurs only at high-symmetry points of Brillouin zone. For TMDs, such conditions are present at the $\mathrm{K}$ point of Brillouin zone (see Fig. 2), and therefore, strong A and B transitions are observed in absorption spectra, see for example, absorption spectra for $\mathrm{MoS}_{2}$ in Refs. 5 and 13. However, very similar conditions are present at the $\mathrm{H}$ point of Brillouin zone, see Fig. 2, but optical transitions at these points were not yet identified in absorption spectra measured for these crystals. Regarding the intensity of optical transitions, a direct application of Fermi's Golden Rule to compare this intensity at different CPs of Brillouin zone does not work, especially in modulation spectroscopy where this intensity also depends on the sensitivity of a given CP to the modulated parameter, i.e., this sensitivity will be different for optical transitions at different CPs of Brillouin zone.

According to our calculations, the energy difference between $\mathrm{A}$ and $\mathrm{A}_{\mathrm{H}}$ transition is rather low and appear in the valence band while the position of conduction band at $\mathrm{K}$ and $\mathrm{H}$ point is almost the same. For the second valence band (see transition labeled as B in Fig. 2), the difference between valence band maximum at the $\mathrm{K}$ and $\mathrm{H}$ point is almost negligible for the four crystals. It means that the $\mathrm{B}$ and $\mathrm{B}_{\mathrm{H}}$ transitions are extremely hard to be resolved in absorption spectra or even in modulated absorption (e.g., PR) which spectrally is more sensitive due to its differential character. However, $\mathrm{A}$ and $\mathrm{A}_{\mathrm{H}}$ transitions can be distinguished in modulated reflectance spectra and such two transitions were recently identified in PR spectra of $\mathrm{MoS}_{2}{ }^{1}{ }^{1}$ In order to study optical transitions at $\mathrm{K}$ and $\mathrm{H}$ points of Brillouin zone for the four crystals, we applied CER, PR, and PzR spectroscopy. Due to a differential character, the spectral resolution of modulated reflectance is better than the resolution of regular absorption, and therefore, $\mathrm{A}$ and $\mathrm{A}_{\mathrm{H}}$ transition can be resolved in CER, $\mathrm{PR}$, and PzR spectra.

The left panel in Fig. 3 shows CER, PR, and PzR spectra measured for $\mathrm{MoS}_{2}$ crystal in the spectral range of $\mathrm{A}$ and $\mathrm{B}$ transitions at room temperature. In the vicinity of the A transition, two resonances are clearly visible. Two resonances can also be present in the vicinity of B transitions, but they cannot be clearly separated, and therefore, a single resonance is used to simulate $\Delta \mathrm{R} / \mathrm{R}$ spectra in this spectral region.

In order to determine the energies of the optical transitions from CER, $P R$, and PzR measurements, the $\triangle R / R$ spectra were analyzed using the standard $\mathrm{CP}$ model.$^{38}$ According to this model, a modulated reflectance spectrum can be fitted using the following formula:

$$
\frac{\Delta R}{R}=\operatorname{Re}\left[\sum_{j=1}^{n} C_{j} e^{i \theta_{j}}\left(\hbar \omega-E_{j}+i \Gamma_{j}\right)^{-m_{j}}\right],
$$

where $n$ is the number of the spectral functions to be fitted, $\hbar \omega$ is the photon energy of the probe beam, $E_{j}$ is the CP energy, and $\Gamma_{j}, C_{j}$, and $\theta_{j}$ are the broadening, amplitude, and phase angle, respectively. The term $m_{j}$ refers to the type of CPs, i.e., the nature of optical transitions. In this case, we expect excitonic transition even at room temperature due to large exciton binding energy in this material system. ${ }^{12,39}$ For such transitions, $m=2$.

CER, PR, and PzR spectra obtained for $\mathrm{MoS}_{2}$ crystal can be fitted by three resonances related to excitonic transitions: two at $\mathrm{K}$ point ( $\mathrm{A}$ and $\mathrm{B}$ transition) and one at $\mathrm{H}$ point ( $A_{H}$ transition). The $B_{H}$ transition overlaps spectrally with the $\mathrm{B}$ transition, and therefore, the two transitions are simulated by a single resonance. Fits of $\Delta R / R$ spectra are shown by thick dashed lines in Fig. 3 together with the moduli of the individual CER resonances $(\rho)$, which have been obtained according to Eq. (4)

$$
\Delta \rho_{j}(E)=\frac{\left|C_{j}\right|}{\left[\left(\hbar \omega-E_{j}\right)^{2}+\Gamma_{j}^{2}\right]^{\frac{m_{j}}{2}}},
$$

with parameters derived from the fit. The moduli are shown by color thin solid lines in Fig. 3. A comparison of the moduli of individual resonances allows us to evaluate the accuracy of fitting as well as spectral position, broadening, and
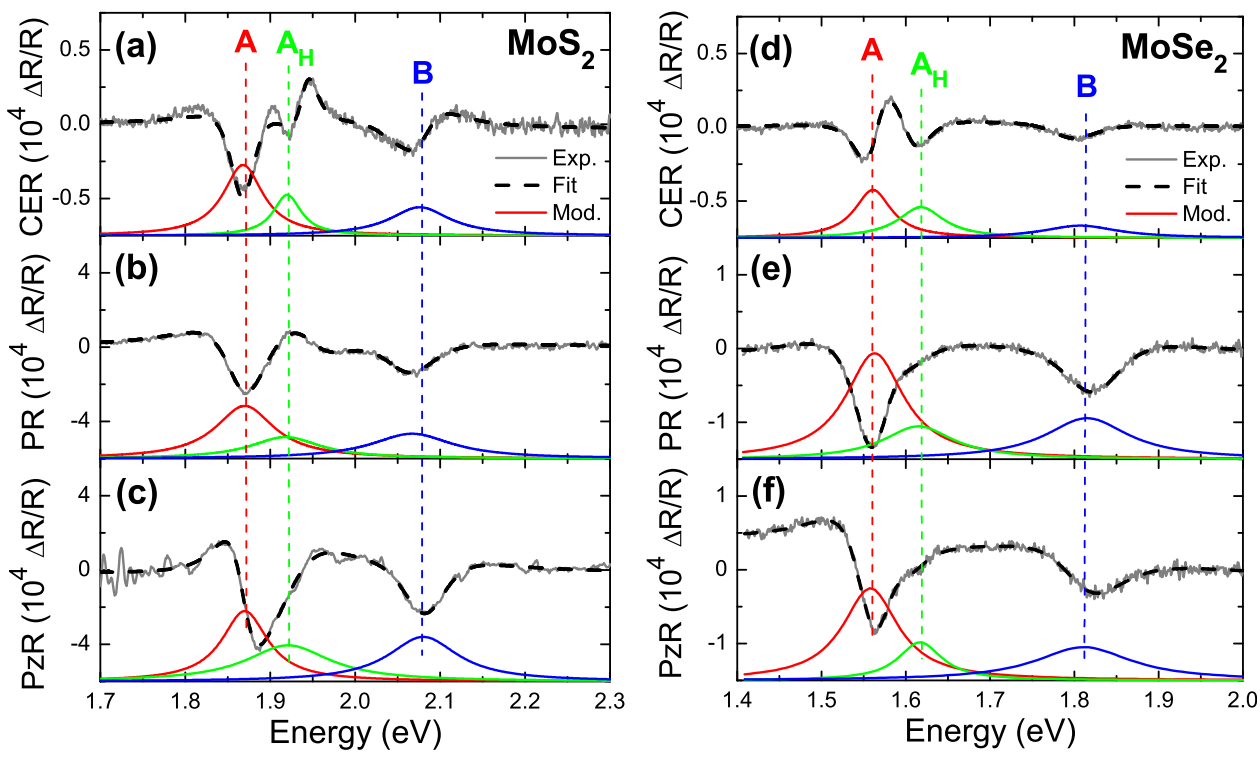

FIG. 3. Room temperature CER, PR, and PzR spectra (grey lines) of bulk $\mathrm{MoS}_{2}$ (left panel) and $\mathrm{MoSe}_{2}$ (right panel) together with fitting curves (thick dashed lines) and their decomposition for individual moduli of $\Delta R / R$ resonances (thin solid color lines). 
intensity of individual optical transitions. The most important parameters, i.e., energies of $\mathrm{A}, \mathrm{A}_{\mathrm{H}}$, and $\mathrm{B}$ transitions, are given in Table I together with the energy separation between these transitions derived from CER, PR, and PzR measurements as well as theoretical predictions. In addition, energies of $\mathrm{A}, \mathrm{A}_{\mathrm{H}}, \mathrm{B}$, and $\mathrm{B}_{\mathrm{H}}$ transitions obtained by PR spectroscopy at low temperatures by Saigal and Ghosh ${ }^{15}$ are given in this table. It is visible that the absolute value of energies of optical transitions extracted from modulated reflectance measurements differ a little from theoretical predictions, but it is a rather well known problem in DFT calculations and should be of no concern. The overall shape of the band structure and the relative changes in the band structures should be perfectly correct, since the MBJLDA functional applied in the calculations significantly improves the results over standard LDA/GGA methods. ${ }^{29,30}$ It means that the absolute values of energies of direct optical transitions in TMDs can be overestimated but relative changes in the electronic band structure of TMDs (i.e., the energy difference between $\mathrm{A}$ and $\mathrm{A}_{\mathrm{H}}$ transition) should be, and in fact are, close to experimental data. Taking into account the above argument, we can compare the energy difference between the $\mathrm{A}$ and $\mathrm{A}_{\mathrm{H}}$ transition obtained from DFT calculations with those derived from modulated reflectance measurements, see Table I. Theoretical prediction gives the value of $33 \mathrm{meV}$. Experimental data are very close to this value and equal 51,48 , and $51 \mathrm{meV}$ for CER, PR, and PzR measurements. It is worth noting that the energy difference between the $\mathrm{B}$ and $\mathrm{B}_{\mathrm{H}}$ transition is negative and about twice smaller than the difference between $\mathrm{A}$ and $\mathrm{A}_{\mathrm{H}}$ transition. Because of this, the two transitions (B and $\mathrm{B}_{\mathrm{H}}$ ) are not resolved in modulated reflectance. The work made by
Saigal and Ghosh was an inspiration to pursue the issue of possible transitions in the other plane of the $\mathrm{BZ}$ where the $\mathrm{H}$ point is located. The measurements performed there seem to be in excellent quality and agree with both our experimental and theoretical results. Due to the low temperature of their measurements, the authors were also able to distinguish the very spectrally close $\mathrm{B}$ and $\mathrm{B}_{\mathrm{H}}$ transitions, only about $20 \mathrm{meV}$ apart, which is extremely challenging. However, in light of our current theoretical calculations, the band structures clearly show that the $B_{H}$ transition should be energetically lower than the $\mathrm{B}$ transition, unlike in the case of the $\mathrm{A} / \mathrm{A}_{\mathrm{H}}$ transitions. Due to the lack of proper theoretical calculations, the authors of Ref. 15 could not predict that fact and seem to misinterpret those transitions, and in fact, the $\mathrm{B}_{\mathrm{H}}$ and $\mathrm{B}$ transitions should be swapped. This is clearly visible in Table I, where the calculated difference $\Delta \mathrm{B}$ is negative and the experimental value is nearly identical but the sign is opposite. After careful consideration of both our experimental and theoretical results, we suggest that if a pair of the second, energetically higher, transitions can be distinguished, the first, weaker one should be associated with $\mathrm{B}_{\mathrm{H}}$ transition and the second stronger one, higher on the energy scale, is the $\mathrm{B}$ transition at $\mathrm{K}$ point.

The right panel in Fig. 3 shows CER, PR, and PzR spectra measured for $\mathrm{MoSe}_{2}$ crystal in the spectral range of $\mathrm{A}$ and $\mathrm{B}$ transitions at room temperature. In this case, an extra $\mathrm{A}_{\mathrm{H}}$ transition is also observed besides $\mathrm{A}$ and $\mathrm{B}$ transitions. According to our DFT calculations, the energy separation between $\mathrm{A}$ and $\mathrm{A}_{\mathrm{H}}$ transition is about two times larger than in $\mathrm{MoS}_{2}$ and equals $59 \mathrm{meV}$. This prediction agrees perfectly with our experimental data, see Table $\mathrm{I}$. $\mathrm{B}_{\mathrm{H}}$ transition is still

TABLE I. Energies of A, $A_{H}, B$, and $B_{H}$ transitions obtained from DFT calculations and derived from modulated reflectance measurements. $\Delta A_{K H}$ is the energy difference between $A_{H}$ and $A$ transition, $\Delta B_{K H}$ is the energy difference between $B_{H}$ and $A$ transition, and $\Delta A_{A B}$ is the energy difference between $A$ and B transitions.

\begin{tabular}{|c|c|c|c|c|c|c|c|}
\hline & A transition $(\mathrm{eV})$ & $\mathrm{A}_{\mathrm{H}}$ transition $(\mathrm{eV})$ & $\Delta \mathrm{A}_{\mathrm{KH}}(\mathrm{meV})$ & B transition $(\mathrm{eV})$ & $\mathrm{B}_{\mathrm{H}}$ transition $(\mathrm{eV})$ & $\Delta \mathrm{B}_{\mathrm{KH}}(\mathrm{meV})$ & $\Delta \mathrm{E}_{\mathrm{AB}}(\mathrm{meV})$ \\
\hline \multicolumn{8}{|l|}{$\mathrm{MoS}_{2}$} \\
\hline DFT & 1.641 & 1.674 & 33 & 1.838 & 1.821 & -17 & 196 \\
\hline CER & 1.869 & 1.920 & 51 & 2.076 & NA & NA & 207 \\
\hline PR & 1.870 & 1.918 & 48 & 2.067 & NA & NA & 197 \\
\hline PzR & 1.870 & 1.921 & 51 & 2.080 & NA & NA & 210 \\
\hline $\mathrm{PR}^{15}$ & 1.941 & 1.973 & 32 & 2.151 & 2.171 & 20 & 210 \\
\hline \multicolumn{8}{|c|}{$\mathrm{MoSe}_{2}$} \\
\hline DFT & 1.381 & 1.440 & 59 & 1.632 & 1.616 & -16 & 251 \\
\hline CER & 1.561 & 1.618 & 57 & 1.807 & NA & NA & 246 \\
\hline PR & 1.563 & 1.615 & 52 & 1.815 & NA & NA & 252 \\
\hline PzR & 1.558 & 1.617 & 59 & 1.811 & NA & NA & 253 \\
\hline \multicolumn{8}{|l|}{$\mathrm{WS}_{2}$} \\
\hline DFT & 1.698 & 1.729 & 31 & 2.140 & 2.135 & -5 & 442 \\
\hline CER & 1.998 & 2.049 & 51 & 2.446 & NA & NA & 448 \\
\hline PR & 1.996 & 2.048 & 52 & 2.438 & NA & NA & 442 \\
\hline PzR & 1.997 & 2.030 & 33 & 2.425 & NA & NA & 428 \\
\hline \multicolumn{8}{|l|}{$\mathrm{WSe}_{2}$} \\
\hline DFT & 1.392 & 1.449 & 57 & 1.884 & 1.882 & -2 & 492 \\
\hline CER & 1.650 & 1.702 & 52 & 2.100 & NA & NA & 450 \\
\hline PR & 1.653 & 1.696 & 43 & 2.091 & NA & NA & 438 \\
\hline PzR & 1.656 & 1.703 & 47 & 2.093 & NA & NA & 437 \\
\hline
\end{tabular}


not clearly resolved in modulated reflectance spectra despite the fact that the energy separation between $\mathrm{B}$ and $\mathrm{B}_{\mathrm{H}}$ transition is about two times larger than in $\mathrm{MoS}_{2}$.

CER, PR, and PzR spectra for the remaining two crystals $\left(\mathrm{WS}_{2}\right.$ and $\left.\mathrm{WSe}_{2}\right)$ are shown in Fig. 4. For $\mathrm{WS}_{2}$ sample, $A$ and $A_{H}$ transitions are very clearly visible in CER and PR spectrum. In PzR spectrum, they are also visible but they are more weakly resolved. The energy separation between $\mathrm{A}$ and $\mathrm{A}_{\mathrm{H}}$ transition is close to theoretical prediction $(51,52$, and $33 \mathrm{meV}$ vs $31 \mathrm{meV}$ ). According to our calculations, $\mathrm{B}$ and $\mathrm{B}_{\mathrm{H}}$ transitions are basically identical, separated by only -5 and $-2 \mathrm{meV}$ (in $\mathrm{WS}_{2}$ and $\mathrm{WSe}_{2}$, respectively), and therefore, they are not resolved in modulated reflectance spectra, and a single resonance is used to fit these spectra in this spectral range.

For $\mathrm{WSe}_{2}$ sample, two resonances are used to fit $\Delta \mathrm{R} / \mathrm{R}$ spectra in the region of $\mathrm{A} / \mathrm{A}_{\mathrm{H}}$ transitions, and they reproduce experimental data very well. However, it is worth noting that the conduction band in this crystal is significantly splitted at both $\mathrm{K}$ and $\mathrm{H}$ points. This issue was not commented for previous samples $\left(\mathrm{MoS}_{2}, \mathrm{MoSe}_{2}\right.$, and $\mathrm{WS}_{2}$ crystals $)$ since this splitting was small $(<2 \mathrm{meV}$ at $\mathrm{K}$ point and $<3 \mathrm{meV}$ at $\mathrm{H}$ point for $\mathrm{MoS}_{2} ;<18 \mathrm{meV}$ at $\mathrm{K}$ point and $<22 \mathrm{meV}$ at $\mathrm{H}$ point for $\mathrm{MoSe}_{2}$; $<32 \mathrm{meV}$ at $\mathrm{K}$ point and $<27 \mathrm{meV}$ at $\mathrm{H}$ point for $\mathrm{WS}_{2}$ ). For $\mathrm{WSe}_{2}$, this splitting equals $49 \mathrm{meV}$ at $\mathrm{K}$ point and $35 \mathrm{meV}$ at $\mathrm{H}$ point. It means that the optical transition between the top valence band and the second conduction band at the $\mathrm{K}$ point of Brillouin zone is expected to be $49 \mathrm{meV}$ above the A transition, and overlaps spectrally with $\mathrm{A}_{\mathrm{H}}$ transition. Since modulated reflectance spectra are very well simulated by two resonances in the region of $\mathrm{A} / \mathrm{A}_{\mathrm{H}}$ transitions, it is rather not justified to use an extra resonance to simulate $\Delta R / R$ spectra in this spectral range. However, we have to be aware that the splitting in conduction band leads to extra transitions in modulated reflectance spectra or larger broadening of optical transitions. In the spectral range of $\mathrm{B} / \mathrm{B}_{\mathrm{H}}$ transition, two resonances are clearly observed in PR spectrum. However, one of them is rather related to an optical transition at band nesting ${ }^{36}$ than an optical transition at $\mathrm{H}$ point. According to our calculations, the energy difference between $\mathrm{B}$ and $\mathrm{B}_{\mathrm{H}}$ transition is small $(-2 \mathrm{meV})$, and therefore, a single resonance was used to simulate this transition. For the PR spectrum, an extra resonance has been added to simulate the optical transition at the nesting point of Brillouin zone. According to our calculations of the electronic band structure for this crystal, it could be an optical transition near the $\mathrm{K}$ point of the Brillouin zone, see transition labeled as $A_{\Lambda}$ in Fig. 2(d). According to our calculations, $A_{\Lambda}$ transition is expected to be $\sim 120-160 \mathrm{meV}$ above the $\mathrm{B}$ transition if we neglect excitonic effects. It is very consistent with our experimental data, see PR spectrum in Fig. 4(e), where an overlap between resonances related to B and $\mathrm{A}_{\Lambda}$ transition is visible and the energy separation between these resonances is $\sim 110 \mathrm{meV}$. It is worth noting that band nesting is also present for remaining TMDs, but optical transitions at these points are expected at higher energies than $\mathrm{B}$ transitions, and therefore, they are not observed in our $\Delta R / R$ spectra in the spectral range discussed in this paper. Optical transitions at band nesting in $\mathrm{MoS}_{2}, \mathrm{MoSe}_{2}, \mathrm{WS}_{2}$, and $\mathrm{WSe}_{2}$ crystals will be discussed elsewhere.

Summarizing results of modulated reflectance measurements for $\mathrm{MoS}_{2}, \mathrm{MoSe}_{2}, \mathrm{WS}_{2}$, and $\mathrm{WSe}_{2}$ crystals, it is clear that an extra resonance is observed a few tens of meV above the A transition for all four crystals. This transition can be attributed to a direct optical transition at the $\mathrm{H}$ point of Brillouin zone $\left(\mathrm{A}_{\mathrm{H}}\right.$ transition), since a significant critical point is expected there, according to our DFT calculations. In addition, $a \mathrm{~B}_{\mathrm{H}}$ transition is expected at almost the same energy as the B transition, but the two transitions are not resolved in our modulated reflectance spectra due to this small energy separation between them. The small energy separation between optical transitions at $\mathrm{K}$ and $\mathrm{H}$ point can be the reason why the optical transitions at $\mathrm{H}$ point of Brillouin zone are usually neglected when the optical absorption spectra are analyzed. The other reason can be excited states of excitonic transitions. They are usually expected also a few tens of meV above $\mathrm{A}$ and $\mathrm{B}$ transitions which may cause the confusion. In general, a signal which could be
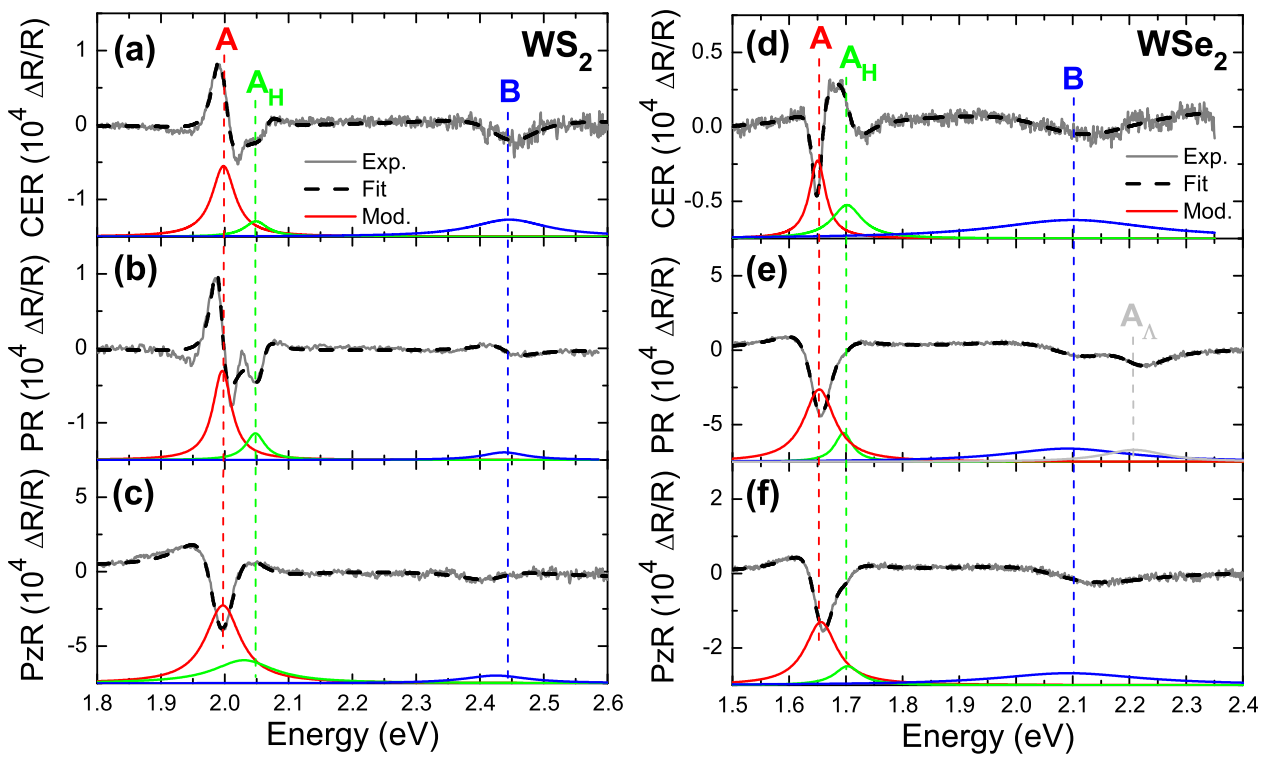

FIG. 4. Room temperature CER, PR, and PzR spectra (grey lines) of bulk $\mathrm{WS}_{2}$ (left panel) and $\mathrm{WSe}_{2}$ (right panel) together with fitting curves (thick dashed lines) and their decomposition for individual moduli of $\Delta \mathrm{R} / \mathrm{R}$ resonances (thin solid color lines). 
related to excited states of $\mathrm{A}$ and $\mathrm{B}$ excitonic transition can be present in $\Delta R / R$ spectra, but the $A_{H}$ resonances we observe are too strong to be attributed to an excited state of the A exciton. We expect that $\Delta R / R$ signal, which could be related to excited states of $\mathrm{A}$ excitonic transition, is very weak at room temperature, and therefore, such transition can be neglected in our analysis of $\Delta R / R$ signal while the $A_{H}$ transition is relatively strong due to the presence of a critical point at the $\mathrm{H}$ point of Brillouin zone very similar to the one at the $\mathrm{K}$ point of Brillouin zone. Therefore, optical transitions at the $\mathrm{H}$ point of Brillouin zone are observed in $\Delta \mathrm{R} / \mathrm{R}$ spectra, besides optical transitions at $\mathrm{K}$ point.

\section{CONCLUSIONS}

It has been shown that the electronic band structure at the $\mathrm{H}$ point of Brillouin zone is very symmetric and similar to the electronic band structure at $\mathrm{K}$ point, and therefore, direct optical transitions at $\mathrm{H}$ point should be expected in modulated reflectance spectra besides direct optical transitions at $\mathrm{K}$ point of Brillouin zone. The direct optical transition at $\mathrm{H}$ point $\left(\mathrm{A}_{\mathrm{H}}\right.$ transition) has been observed a few tens of meV above the A transition in CER, PR, and PzR spectra for the four crystals. The second transition at the $\mathrm{H}$ point of Brillouin zone $\left(\mathrm{B}_{\mathrm{H}}\right.$ transition) overlaps spectrally with the $\mathrm{B}$ transition at the $\mathrm{K}$ point, and therefore, an extra resonance which could be related to the $\mathrm{B}_{\mathrm{H}}$ transition is not resolved in modulated reflectance spectra at room temperature. However, the theoretical calculations show that the $\mathrm{B}_{\mathrm{H}}$ transition should be expected for slightly lower energies than the $\mathrm{B}$ transition, in contrast to $\mathrm{A}_{\mathrm{H}}$ and $\mathrm{A}$, which is especially important in $\mathrm{MoS}_{2}$ where the $\mathrm{B}-\mathrm{B}_{\mathrm{H}}$ separation is the largest. The spectral difference between $A$ and $A_{H}$ transitions has been found to be in a very good agreement with theoretical predictions.

\section{ACKNOWLEDGMENTS}

The DFT calculations were performed in the Wroclaw Centre for Networking and Supercomputing. M.P.P. acknowledges the support within the "Diamond Grant" (DI2013 006143) from the MNiSzW.

${ }^{1}$ Q. Tang and A. Zhou, "Graphene-analogous low-dimensional materials," Prog. Mater. Sci. 58, 1244 (2013).

${ }^{2}$ H. Wang, H. Yuan, S. S. Hong, Y. Li, and Y. Cui, "Physical and chemical tuning of two-dimensional transition metal dichalcogenides," Chem. Soc. Rev. 44, 2664 (2015).

${ }^{3}$ T. Heine, "Transition metal chalcogenides: ultrathin inorganic materials with tunable electronic properties," Acc. Chem. Res. 48, 65 (2015).

${ }^{4}$ J. A. Wilson and A. D. Yoffe, "The transition metal dichalcogenides discussion and interpretation of the observed optical, electrical and structural properties," Adv. Phys. 18, 193 (1969).

${ }^{5}$ A. R. Beal, J. C. Knights, and W. Y. Liang, "Transmission spectra of some transition metal dichalcogenides. II. Group VIA: trigonal prismatic coordination,” J. Phys. C: Solid State Phys. 5, 3540 (1972).

${ }^{6} \mathrm{~L}$. F. Mattheiss, "Band structures of transition-metal-dichalcogenide layer compounds," Phys. Rev. B 8, 3719 (1973).

${ }^{7}$ R. V. Kasowski, "Band structure of $\mathrm{MoS}_{2}$ and $\mathrm{NbS}_{2}$," Phys. Rev. Lett. 30, 1175 (1973).

${ }^{8}$ R. Coehoorn, C. Haas, J. Dijkstra, C. J. F. Flipse, R. A. de Groot, and A. Wold, "Electronic structure of $\mathrm{MoSe}_{2}, \mathrm{MoS}_{2}$, and $\mathrm{WSe}_{2}$. I. Band-structure calculations and photoelectron spectroscopy," Phys. Rev. B 35, 6195 (1987).
${ }^{9}$ R. Mamy, A. Boufelja, and B. Carricaburu, "Angle resolved photoemission and electronic band structure of $\mathrm{MoS}_{2}$," Phys. Status Solidi B 141, 467 (1987).

${ }^{10}$ K. Fives, I. T. McGovern, R. McGrath, R. Cimino, G. Hughes, A. McKinley, and G. Thornton, "The photoelectron bandstructure of molybdenum disulphide," J. Phys.: Condens. Matter 4, 5639 (1992).

${ }^{11} \mathrm{~K}$. Kobayashi and J. Yamauchi, "Electronic structure and scanning-tunnelingmicroscopy image of molybdenum dichalcogenide surfaces," Phys. Rev. B 51, 17085 (1995).

${ }^{12}$ T. Cheiwchanchamnangij and W. R. L. Lambrecht, "Quasiparticle band structure calculation of monolayer, bilayer, and bulk $\mathrm{MoS}_{2}$," Phys. Rev. B 85, 205302 (2012).

${ }^{13}$ A. Molina-Sanchez, D. Sangalli, K. Hummer, A. Marini, and L. Wirtz, "Effect of spin-orbit interaction on the optical spectra of single-layer, double-layer, and bulk MoS 2 ," Phys. Rev. B 88, 045412 (2013).

${ }^{14}$ R. Roldán, J. A. Silva-Guillén, M. Pilar López-Sancho, F. Guinea, E. Cappelluti, and P. Ordejón, "Electronic properties of single-layer and multilayer transition metal dichalcogenides $\mathrm{MX}_{2}(\mathrm{M}=\mathrm{Mo}, \mathrm{W}$ and $\mathrm{X}=\mathrm{S}$, Se)," Ann. Phys. (Berlin) 526, 347 (2014).

${ }^{15}$ N. Saigal and S. Ghosh, "H-point exciton transitions in bulk $\mathrm{MoS}_{2}$," Appl. Phys. Lett. 106, 182103 (2015).

${ }^{16}$ J. Bordas and E. A. Davi, "Electromodulation spectroscopy of excitons: molybdenum disulphide," Phys. Status Solidi B 60, 505 (1973).

${ }^{17}$ E. Fortin and F. Raga, "Excitons in molybdenum disulphide," Phys. Rev. B 11, 905 (1975).

${ }^{18} \mathrm{H}$. Meinhold and G. Weiser, "Modulation spectroscopy on $\mathrm{MoS}_{2}$, and $\mathrm{MoSe}_{2}$," Phys. Status Solidi B 73, 105 (1976).

${ }^{19}$ K. Saiki, M. Yoshimi, and S. Tanaka, "Modulation spectroscopy on the group IV and VI transition-metal dichalcogenides," Phys. Status Solidi B 88, 607 (1978).

${ }^{20}$ K. K. Tiong, T. S. Shou, and C. H. Ho, "Temperature dependence piezoreflectance study of the effect of doping $\mathrm{MoS}_{2}$ with rhenium," J. Phys.: Condens. Matter 12, 3441 (2000).

${ }^{21}$ P. C. Yen, H. P. Hsu, Y. T. Liu, Y. S. Huang, and K. K. Tiong, "Temperature dependences of energies and broadening parameters of the band-edge excitons of Re-doped $\mathrm{WS}_{2}$ and $2 \mathrm{H}-\mathrm{WS}_{2}$ single crystals," J. Phys.: Condens. Matter 16, 6995 (2004).

${ }^{22}$ D. O. Dumcenco, H. P. Hsu, Y. S. Huang, C. H. Liang, K. K. Tiong, and C. H. Du, "Optical properties of tungsten disulfide single crystals doped with gold," Mater. Chem. Phys. 111, 475 (2008).

${ }^{23}$ Y. J. Wu, P. H. Wu, J. Jadczak, Y. S. Huang, C. H. Ho, H. P. Hsu, and K. $\mathrm{K}$. Tiong, "Piezoreflectance study of near band edge excitonic-transitions of mixed-layered crystal $\mathrm{Mo}\left(\mathrm{S}_{\mathrm{x}} \mathrm{Se}_{1-\mathrm{x}}\right)_{2}$ solid solutions," J. Appl. Phys. 115, 223508 (2014).

${ }^{24}$ M. Sigiro, Y.-S. Huang, Ch.-H. Ho, Y.-Ch. Lin, and K. Suenaga, "Influence of rhenium on the structural and optical properties of molybdenum disulfide,” Jpn. J. Appl. Phys., Part 1 54, 04DH05 (2015).

${ }^{25}$ P. Blaha, K. Schwarz, G. Madsen, D. Kvasnicka, and J. Luitz, WIEN2k, An Augmented Plane Wave + Local Orbitals Program for Calculating Crystal Properties (Karlheinz Schwarz, Techn. Universität Wien, Austria, 2001).

${ }^{26}$ S. Grimme, J. Antony, S. Ehrlich, and H. Krieg, "A consistent and accurate ab initio parametrization of density functional dispersion correction (DFT-D) for the 94 elements H-Pu," J. Chem. Phys. 132, 154104 (2010).

${ }^{27}$ J. P. Perdew, K. Burke, and M. Ernzerhof, "Generalized gradient approximation made simple,” Phys. Rev. Lett. 77, 3865 (1996).

${ }^{28}$ F. Dybała, M. Polak, J. Kopaczek, P. Scharoch, S. Tongay, and R. Kudrawiec, "Pressure coefficients for direct optical transitions in $\mathrm{MoS}_{2}$, $\mathrm{MoSe}_{2}, \mathrm{WS}_{2}$, and $\mathrm{WSe}_{2}$ crystals and semiconductor to metal transition," Sci. Rep. 6, 26663 (2016).

${ }^{29}$ F. Tran and P. Blaha, "Accurate band gaps of semiconductors and insulators with a semilocal exchange-correlation potential," Phys. Rev. Lett. 102, 226401 (2009).

${ }^{30}$ J. A. Camargo-Martínez and R. Baquero, "Performance of the modified Becke-Johnson potential for semiconductors," Phys. Rev. B 86, 195106 (2012).

${ }^{31}$ M. P. Polak, P. Scharoch, and R. Kudrawiec, "First-principles calculations of bismuth induced changes in the band structure of dilute $\mathrm{Ga}-\mathrm{V}-\mathrm{Bi}$ and In-V-Bi alloys: chemical trends versus experimental data," Semicond. Sci. Technol. 30, 094001 (2015).

${ }^{32}$ M. P. Polak, P. Scharoch, R. Kudrawiec, J. Kopaczek, M. J. Winiarski, W. M. Linhart, M. K. Rajpalke, K. M. Yu, T. S. Jones, M J. Ashwin, and 
T. D. Veal, "Theoretical and experimental studies of electronic band structure for $\mathrm{GaSb}_{1-\mathrm{x}} \mathrm{Bi}_{\mathrm{x}}$ in the dilute Bi regime," J. Phys. D 47, 355107 (2014).

${ }^{33}$ R. Kudrawiec and J. Misiewicz, "Photoreflectance and contactless electroreflectance measurements of semiconductor structures by using bright and dark configurations," Rev. Sci. Instrum. 80, 096103 (2009).

${ }^{34}$ R. Kudrawiec, "Application of contactless electroreflectance to III-nitrides," Phys. Status Solidi B 247, 1616 (2010).

${ }^{35}$ M. Grundmann, The Physics of Semiconductors: An Introduction Including Nanophysics and Applications (Springer-Verlag, Berlin, Germany, 2006).
${ }^{36}$ A. Carvalho, R. M. Ribeiro, and A. H. C. Neto, "Band nesting and the optical response of two-dimensional semiconducting transition metal dichalcogenides," Phys. Rev. B 88, 115205 (2013).

${ }^{37}$ F. Bassani and G. Pastori Parravicini, Electronic States and Optical Transitions in Solids (Pergamon Press, 1975).

${ }^{38}$ D. E. Aspnes, "Third-derivative modulation spectroscopy with low-field electroreflectance," Surf. Sci. 37, 418 (1973).

${ }^{39}$ T. Goto, Y. Kato, K. Uchida, and N. Miura, "Exciton absorption spectra of $\mathrm{MoS}_{2}$ crystals in high magnetic fields up to $150 \mathrm{~T}$," J. Phys.: Condens. Matter 12, 6719 (2000). 